\section{Birgitt Holschuh-Lorang}

Das Bild der Angst bei Kindern und Jugendlichen ist vielfältig. Sie klagen über Körpersymptome wie Kopfschmerzen und Konzentrationsstörungen, Übelkeit mit Brechreiz, Bauchkrämpfe oder Parästhesien. Die Körpersymptome sind in der Regel nicht organischen Ursprungs, wie gründliche körperliche Untersuchungen beim Kinderarzt ergeben.

Die Kinder können aber auch Ängste äussern, die teilweise regelrecht panisch sind, jedoch keinen realen Bezug aufweisen. Sie klagen über Aufgeregtsein, das das normale Mass überschreitet, und innere Unruhe bei Klassenarbeiten sowie bisweilen über eine regelrechte Schulphobie. Zusammen mit Konzentrationsstörungen können diese Symptome zu eingeschränkten Schulleistungen führen.

Erwartungshaltungen von Eltern werden somit nicht erfüllt und der innere Druck auf das Kind steigt zunehmend. Kinderpsychologische Betreuung und Hausaufgabenhilfe bringen nicht immer die gewünschte Wirkung, sodass Eltern bisweilen mit Überforderung auf das Verhalten ihrer Kinder reagieren.

\section{Lösungsansätze durch Aufdecken von Energieblockaden}

In meiner naturheilkundlichen Praxis liegt der diagnostische und therapeutische Schwerpunkt in einer anderen Betrachtungsweise von körperlichen und seelischen Störungen. Nach Sichtweise der Psychosomatischen Energetik (PSE), einem Diagnostik- und Therapieverfahren, das von Dr. Reimar Banis entwickelt wurde, verbindet die feinstoffliche Energie die körperliche mit der seelischen

\title{
Angststörungen bei Kindern
}

Ebene. Diese feinstoffliche Energie wiederum beeinflusst unser vegetatives Nervensystem, dessen Steuerimpulse für ein gutes Funktionieren der Organtätigkeit sorgen. Ist dieses feinstoffliche Energiefeld gestört, weil Aufnahme und Fluss der Energie blockiert sind, erklären sich auf diese Weise die oben angeführten Körpersymptome.

Unser Gesundsein und Wohlbefinden wird nicht nur durch eine intakte Körperebene bestimmt; auch unsere seelischen Funktionen sollten stabil sein. Daher ist auch für diese Ebene ein ausreichendes Mass an Energie notwendig, um gut gestimmt und psychisch belastbar zu sein.

Die Ursachen für die gestörte Energieaufnahme können mithilfe der PSE herausgefunden werden. Es kann zunächst eine Aussage über die aktuelle Befindlichkeit der Kinder getroffen werden, d.h. wie gut ihre körperlich vitale und wie gut ihre seelische Energieladung ist. Dabei orientiert man sich an Normwerten von $100 \%$. In der Regel wird die Energieaufnahme durch seelische Traumen aus der Vergangenheit, die verdrängt wurden, blockiert (siehe Abb. 1 eines Konflikts, der einen Teil der Körperenergie aufgenommen hat). Diese seelischen Energieräuber sind mit speziellen homöopathischen Komplexmitteln auflösbar. Das führt bei den kleinen Patienten zu einem verbesserten körperlichen Befinden und einer stabileren seelischen Belastbarkeit.

Nachfolgend sollen 2 Beispiele aus meiner Praxis die praktische Anwendung der PSE dokumentieren:

\section{Fall 1: Lena, 12 Jahre}

Lena besucht die Erweiterte Realschule und ist bei Klassenarbeiten furchtbar aufgeregt. Das führt bei ihr zu einer starken Einschränkung ihrer Konzentrationsfähigkeit und in der Folge zu schlechteren Schulleistungen. Die Mutter ist besorgt, weiss aber für ihr Kind, mit dem sie lernt und damit versucht, es gut vorzubereiten, keinen Rat.

Bei der ersten Messung zeigt Lena einen deutlich eingeschränkten Wert ihrer emotionalen Belastbarkeit mit 60\% (Normwert 100\%). Die Ursache hierfür liegt in einem Konflikt des
Abb. 1. Ein Konflikt (Kreis) wirkt im feinstofflichen Energiefeld als Energieblockade.

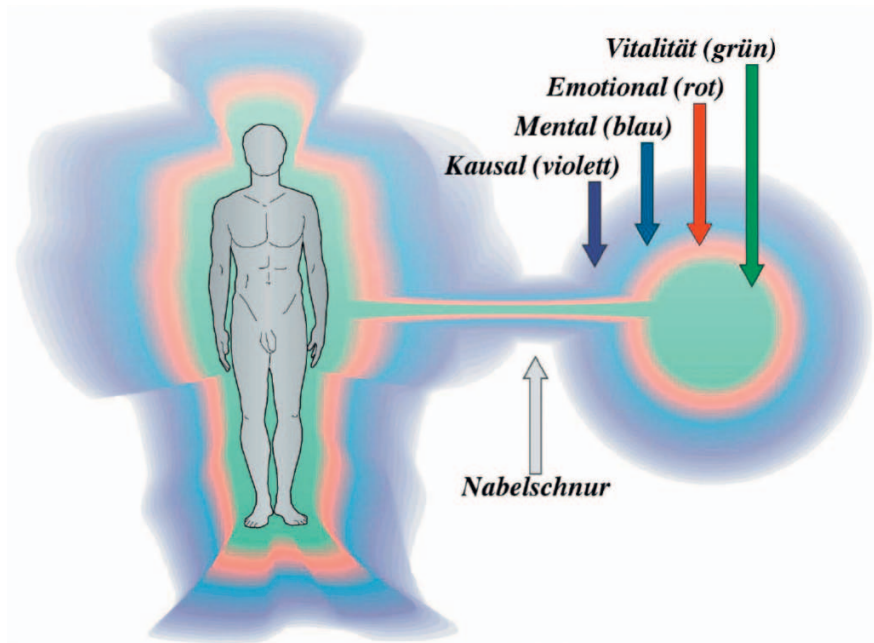

\section{KARGER}

Fax +497614520714 Information@Karger.d www.karger.com
(๑) 2012 S. Karger GmbH, Freiburg
Dr. med. Birgitt Holschuh-Lorang

Fachärztin für Allgemeinmedizin-Naturheilverfahren

Zum Scheidberg 9, 66798 Wallerfangen-Gisingen, Deutschland

Tel. +496837901999 
vegetativen Zentrums im Bereich des Herzens. Dieses Zentrum entspricht nach der altindischen Yogalehre dem 4. oder Herzchakra. Das Konfliktthema, das bei Lena die Energieaufnahme blockiert, ist ein längst vergangenes, verdrängtes Problem in der Vertrauensebene, ein «Verängstigungskonflikt». Es werden die Mittel Chavita 4 und Emvita 15 zur energetischen Auflösung verabreicht.

Nach 3 Monaten Behandlungszeit wird Lena wieder getestet; der Wert der emotionalen Belastbarkeit ist auf $80 \%$ gestiegen. Lena kann sich deutlich besser konzentrieren und ist bei Klassenarbeiten nicht mehr so aufgeregt. Ihre Leistungen haben sich dadurch so deutlich verbessert, dass sie den weiterführenden Zweig dieser Schule besuchen darf.

\section{Fall 2: Viktor, 10 Jahre}

Viktor ist ein Kind mit guten Schulleistungen. Körperlich ist er gesund. Allerdings bestehen seit mehreren Wochen regelrechte Panikattacken. Er äussert stärkste Ängste, klagt über Parästhesien in den Beinen und Übelkeit. Er ist sehr blass. Diese Symptome führen zu einer Schulphobie. Es gibt für diese Symptome weder im häuslichen noch im schulischen Umfeld eine Erklärung. Eine kinderpsychiatrische Behandlung hilft nicht weiter.

Bei der Erstuntersuchung hat Viktor einen sehr stark eingeschränkten Wert der emotionalen Belastbarkeit. Er beträgt nur 20\%.

Auch hier liegt die Ursache in einer gestörten Energieaufnahme im Bereich des Herzchakra. Das dahinter steckende seelische Thema ist ein Panikkonflikt, der mit Chavita 4 und Emvita 16 behandelt wird. Viktor benötigt zusätzlich zur schnelleren Stabilisierung seiner Angstsymptomatik das Mittel Anxiovita.

Ich sehe Viktor 6 Monate später wieder. Der Wert der emotionalen Belastbarkeit ist auf $60 \%$ gestiegen. Es geht ihm deutlich besser; die Körpersymptome sind verschwunden und auch die starken Ängste haben sich deutlich gebessert.

\section{Zusammenfassung}

Diese Fallbeispiele stehen für viele andere und zeigen den grossen Einfluss der feinstofflichen Energie auf unser körperliches Wohlbefinden und auf unsere seelische Gesundheit. Dass die Ursache in tief verborgenen, unbewussten, verdrängten Seelenverletzungen liegt, die Lebensenergie sozusagen «wegvampirisiert» haben (siehe Abb. 1), erklärt, warum sich diese Problematik häufig ohne ersichtlichen äusseren Anlass findet. Mit der Methode der PSE können diese tief liegenden Ursachen jedoch aufgedeckt und auf sehr einfachem Wege völlig nebenwirkungsfrei behandelt werden. Gerade für Kinder stellt die PSE daher ein einfaches Instrument der Gesundheitsförderung dar, damit sie frei von Ängsten einen guten Weg ihrer Persönlichkeitsentwicklung beschreiten und ihr seelisch-geistiges Potenzial nutzen können.

\section{Literatur}

Banis R (ed): Lesebuch der Psychosomatischen Energetik, Band 1-4. Hochheim, Co'Med, 2004 Banis U: Wie wirkt Psychosomatische Energetik? Kirchzarten, VAK, 2010. 